\title{
ON THE CLASS NUMBER OF A RELATIVELY CYCLIC NUMBER FIELD
}

\author{
HIDEO YOKOI
}

To Professor Kiyoshi Noshiro on the occasion of his 60th birthday

\section{Introduction}

Let $l$ be a rational prime. For each $n \geqq 0$, denote by $\zeta_{l^{n}}$ a primitive $l^{n}$-th root of unity and by $\mathbf{Q}\left(\zeta_{l^{n}}\right)$ the cyclotomic field obtained by adjoining $\zeta_{l^{n}}$ to the rational field $\mathbf{Q}$. Then a theorem which was proved by $\mathrm{H}$. Weber ${ }^{11}$ is well known:

TheOREM (H. WeBer). The class number of $\mathbf{Q}\left(\zeta_{2} v\right)$ is odd.

As a generalization of this theorem of Weber, Ph. Furtwängler ${ }^{2)}$ gave :

Theorem (Ph. Furtwängler). The class number of $\mathbf{Q}\left(\zeta_{l^{2}}\right)$ is divisible by the prime $l$ if and only if the class number of $\mathbf{Q}\left(\zeta_{l}\right)$ is divisible by $l$.

Moreover, Ph. Furtwängler ${ }^{3)}$ obtained

Theorem ( $\mathrm{P}_{\mathrm{H}}$. Furtwängler). Let $F$ and $K$ be two subfields of $\mathbf{Q}\left\{\zeta_{N}\right)$. If $F$ is contained in $K$, then the class number of $K$ is divisible by the class number of $F$.

Afterwards, K. Iwasawa' generalized these theorems, and got

Theorem (K. IWASAWA $)^{5)}$. Let $F$ be an algebraic number field, and let $K$ be a finite Galois extension of $F$. Then we have the following facts:

(I) If there exists a prime divisor $P$ of $F$ which is fully ramified in the extension $K / F$, then the class number of $K$ is divisible by the class number of $F$.

(II) If, furthermore, $K / F$ is a cyclic extension of prime power degree $l^{2}$ and

Received February 16, 1966.

1) Cf. H. Weber [21].

2) Cf. $\mathrm{Ph}$. Furtwängler [7].

3) Cf. Ph. Furtwängler [6].

4) Cf. K. Iwasawa [12].

5) This theorem is often referred to e.g. in S.-N.Kuroda [16], K. Iwasawa [14] etc. 
HIDEO YOKOI-

has no ramified prime divisor other than $P$, then conversely the class number of $F$ is divisible by $l$ provided the class number of $K$ is divisible by $l$.

In the present paper, we shall give some results on the ideal class number of a relatively cyclic number field including, in particular, a generalization of the theorem of Iwasawa. We shall first give some preliminaries in $\S 2$. Next we shall consider in $\S 3$ the ideal class group of a relatively cyclic number field, and in $\S 4$ ideal class numbers and unit groups. Finally in $\S 5$ we shall give main theorems which include the theorem of Iwasawa.

\section{§. Notations}

Generally, for an arbitrary abelian group $B$ and its subgroup $B^{\prime}$, the order of $B$ and the index of $B^{\prime}$ in $B$ are denoted by $[B]$ and $\left[B: B^{\prime}\right]$, respectively.

The notations which are used throughout this paper for an arbitrary number field $k$ are :

$E_{k}$ : the group of units in $k$.

$\mathbf{C}_{k}$ : the group of absolute ideal classes in $k$.

$\widetilde{k}:$ the absolute class field of $k$.

$h_{k}$ : the number of absolute ideal classes in $k$.

Let $K / F$ be a Galois extension with finite degree $n$ over an algebraic number field $F$ of finite degree, and $G=G(K / F)$ be the Galois group of $K / F$. "Then, as usual, we shall denote by $H^{r}(G, B)$ or sometimes simply by $H^{r}(B)$ the $r$ dimensional Galois cohomology group of $G$ acting on an abelian group $B$, and by $Q(B)$ the Herbrand quotient of $B$, i.e. $Q(B)=\left[H^{0}(G, B)\right] /\left[H^{1}(G, B)\right]$. Furthermore, we used the notations

$\Pi e(p)$ : product of the ramification exponents of all the finite prime divisors $\mathfrak{p}$ in $F$ with respect to $K / F$.

$\Pi e\left(p_{\infty}\right)$ : product of the ramification exponents of all the infinite prime divisors $\mathfrak{p}_{\infty}$ in $F$ with respect to $K / F$.

$\tilde{n} e(p)$ : product of the ramification exponents of all the finite and infinite prime divisors in $F$ with respect to $K / F$, i.e. $\check{\Pi} e(p)=\Pi e(p) \times \Pi e\left(p_{\infty}\right)$.

$(A)$ : the group of principal ideals in $K$.

(a): the group of principal ideals in $F$.

(є) : the group of units in $F$.

$(\eta)$ : the group of units which are norms of numbers in $K$. 
$\left(A_{0}\right)$ : the group of ambiguous principal ideals in $K / F$.

$\left(a_{F}\right)$ : the group of ideals in $F$.

$\left(a_{0}\right)$ : the group of ambiguous ideals in $K / F$.

A : the group of ambiguous ideal classes in $K / F$.

$\mathbf{A}_{0}$ : the group of ideal classes represented by ambiguous ideals in $K / F$.

$\mathbf{A}_{F}$ : the group of ideal classes of $K$ represented by ideals of $F$.

$N \mathrm{C}_{K}$ : the image by the norm homomorphism of $\mathbf{C}_{K}$ with respect to $K / F$.

${ }_{N} \mathbf{C}_{K}$ : the kernel by the norm homomorphism of $\mathbf{C}_{K}$ with respect to $K / F$.

$a$ : the number of ambiguous ideal classes in $K / F$, i.e. $a=$ [A].

$a_{0}:$ the number of ideal classes represented by ambiguous ideals in $K / F$, i.e. $a_{0}=\left[\mathbf{A}_{0}\right]$.

$h_{0} \quad$ : the number of ideal classes of $F$ which become principal in $K$.

\section{§. Preliminaries}

Let $K / F$ be a Galois extension with finite degree $n$ over an algebraic number field $F$ of finite degree. Then we have the following two lemmas:

Lemma 1.

$$
a_{0}=h_{F} \cdot \frac{\Pi e(\mathfrak{b})}{\left[H^{1}\left(G, E_{K}\right)\right]} \cdot
$$

Proof. For $a_{0}$ we have

$$
a_{0}=\left[\mathbf{A}_{0}\right]=\left[(A)\left(a_{0}\right):(A)\right]=\left[\left(a_{0}\right):\left(A_{0}\right)\right]=\frac{\left[\left(a_{0}\right):(\alpha)\right]}{\left[\left(A_{0}\right):(\alpha)\right]} .
$$

On the other hand, we know that $H^{1}\left(G, E_{K}\right)$ is canonically isomorphic with the factor group of the group of ambiguous principal ideals of $K$ modulo the group of principal ideals of $F^{6)}$, i.e.

$$
H^{1}\left(G, E_{K}\right) \cong\left(A_{0}\right) /(\alpha) \text {. }
$$

Since $\left[\left(a_{0}\right):(\alpha)\right]=\left[\left(a_{0}\right):\left(a_{F}\right)\right]\left[\left(a_{F}\right):(\alpha)\right]=\Pi e(p) \times h_{F}$, lemma 1 is clear.

LEMмA 2. In the following diagram:

6) Cf. K. Iwasawa [13] or A. Brumer-M. Rosen [3]. 


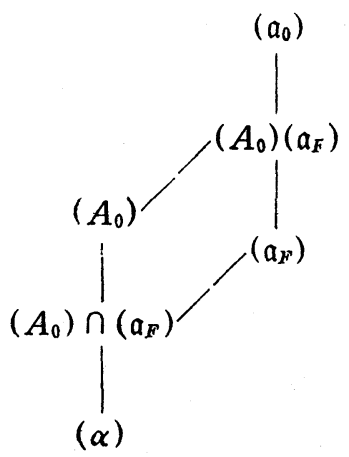

we have

$$
\begin{aligned}
& {\left[\left(A_{0}\right) \cap\left(\mathfrak{a}_{F}\right):(\alpha)\right]=h_{0},} \\
& {\left[\left(\mathfrak{a}_{F}\right):\left(A_{0}\right) \cap\left(\mathfrak{a}_{F}\right)\right]=\left[\left(A_{0}\right)\left(\mathfrak{a}_{F}\right):\left(A_{0}\right)\right]=h_{F} / h_{0},} \\
& {\left[\left(A_{0}\right):\left(A_{0}\right) \cap\left(\mathfrak{a}_{F}\right)\right]=\left[\left(A_{0}\right)\left(\mathfrak{a}_{F}\right):\left(\mathfrak{a}_{F}\right)\right]=\left[H^{1}\left(G, E_{R}\right)\right] / h_{0},} \\
& {\left[\left(\mathfrak{a}_{0}\right):\left(A_{0}\right)\left(\mathfrak{a}_{F}\right)\right]=\operatorname{IIe}(\mathfrak{p}) \cdot h_{0} /\left[H^{1}\left(G, E_{K}\right)\right] .}
\end{aligned}
$$

In particular, $h_{0}$ is a common divisor of $h_{F}$ and $\left[H^{1}\left(G, E_{K}\right)\right]$.

Proof. $\left[\left(A_{0}\right) \cap\left(\mathfrak{a}_{F}\right):(\alpha)\right]=h_{0}$ is a direct consequence of our definition of $h_{0}$. Since $\left[\left(a_{F}\right):(\alpha)\right]=h_{F}$, we have $\left[\left(a_{F}\right):\left(A_{0}\right) \cap\left(a_{F}\right)\right]=h_{F} / h_{v}$, and hence $\left[\left(A_{0}\right)\left(\mathfrak{a}_{F}\right):\left(A_{0}\right)\right]=h_{F} / h_{0}$. On the other hand, since $\left[\left(A_{0}\right):(\alpha)\right]=\left[H^{1}\left(G, E_{K}\right)\right]^{7}$, we have $\left[\left(A_{0}\right):\left(A_{0}\right) \cap\left(a_{F}\right)\right]=\left[H^{1}\left(G, E_{K}\right)\right] / h_{0}$, and hence $\left[\left(A_{0}\right)\left(a_{F}\right):\left(\mathfrak{a}_{F}\right)\right]=$ $\left[H^{1}\left(G, E_{K}\right)\right] / h_{0}$.

Finally, since by lemma 1 we know $\left[\left(a_{0}\right):\left(A_{0}\right)\right]=a_{0}=\Pi e(p) \cdot h_{F} /\left[H^{1}\left(G, E_{K}\right)\right]$, we have $\left[\left(\mathfrak{a}_{0}\right):\left(A_{0}\right)\left(\mathfrak{a}_{F}\right)\right]=\Pi e(\mathfrak{p}) \cdot h_{0} /\left[H^{1}\left(G, E_{K}\right)\right]$.

From now in this $\S$, we suppose especially that $K / F$ is cyclic of finite degree $n$, and let $\sigma$ be a generator of the Galois group $G$.

\section{LEMMA 3.}

$$
Q\left(E_{K}\right)=\Pi e\left(\mathfrak{p}_{\infty}\right) / n^{81} \text { and } Q\left(\mathbf{C}_{K}\right)=1,
$$

namely $\left[H^{r}\left(G, \mathbf{C}_{K}\right)\right]$ is a constant which does not depend on $r$.

Proof. If we let $E_{K}^{\prime}$ be any $G$-subgroup of $E_{K}$ with finite index, then by the lemma of Herbrand we have $Q\left(E_{K}^{\prime}\right)=Q\left(E_{K}\right)$. In particular, we may choose the unit group of $\operatorname{Artin}^{9 \prime}$ as $E_{K}^{\prime}$, and we have $Q\left(E_{K}^{\prime}\right)=\Pi e\left(\mathfrak{p}_{\infty}\right) / n$. Hence we

7) Cf. K. Iwasawa [13] or A. Brumer-M. Rosen [3].

8) Cf. C. Chevalley [5] for the case where $K / F$ is cyclic of prime degree.

9) Cf. E. Artin [2]. 
get $Q\left(E_{K}\right)=\Pi e\left(p_{\infty}\right) / n$.

On the other hand, since $C_{K}$ is a finite $G$-group, we have $Q\left(C_{K}\right)=1$, namely $\left[H^{0}\left(G, \mathbf{C}_{K}\right)\right]=\left[H^{1}\left(G, \mathbf{C}_{K}\right)\right]$, and since $K / F$ is cyclic, we know that $\left[H^{r}\left(G, \mathbf{C}_{K}\right)\right]$ is a constant which does not depend on $r^{10}$.

LEMMA 4. Let $n_{1}, n_{2}$ be invariants of $K / F$ determined by $\frac{h_{F}}{n_{1}}=\left[\mathbf{C}_{K}:{ }_{N} \mathbf{C}_{K}\right]$ and $\frac{\widetilde{M} e(b)}{n_{2} \cdot[\varepsilon: \eta]}=\left[{ }_{N} \mathbf{C}_{K}: \mathbf{C}_{K}^{1-r}\right]=\left[H^{r}\left(G, \mathbf{C}_{K}\right)\right]$ for any integer $r$. Then, for the ambiguous class number $a$, we have $a=\frac{h_{F}}{n_{1}} \times \frac{\tilde{\Pi} e(p)}{n_{2} \cdot[\varepsilon: \eta]} \cdot n_{1} \times n_{2}=n$.

In particular, $h_{F} \times \tilde{n} e(p) \equiv 0 \bmod . n^{11)}$.

Proof. Since $\left[C_{K}:{ }_{N} C_{K}\right]=\left[N C_{K}\right]$ is a divisor of $h_{F}$ and $\left[{ }_{N} C_{K}: C_{K}^{1-0}\right]$ is a divisor of $[(\alpha):(\nu)]=\tilde{\Pi} e(p) /[\varepsilon: \eta]^{12)}$, we may obtain integers $n_{1}, n_{2}$ such that

$$
h_{F}=\left[\mathbf{C}_{K}:{ }_{N} \mathbf{C}_{K}\right] \times n_{1}, \frac{\tilde{n} e(p)}{[\varepsilon: \eta]}=\left[\mathbf{C}_{K}: \mathbf{C}_{K}^{1-a}\right] \times n_{2}
$$

Since $a=[A]=\left[C_{K}: C_{K}^{1-\sigma}\right]=\left[C_{K}:{ }_{N} C_{K}\right]\left[C_{N} C_{K}: C_{K}^{1-\sigma}\right]$, we have

$$
a=\frac{h_{F}}{n_{1}} \times \frac{\widetilde{\Pi} e(\mathfrak{b})}{n_{2} \cdot[\varepsilon: \eta]} .
$$

Furthermore, from lemma 3 we have for any integer $r$

$$
\frac{\hat{\Pi} e(b)}{n_{2} \cdot[\varepsilon: \eta]}=\left[{ }_{N} \mathbf{C}_{K}: \mathbf{C}_{K}^{1-o}\right]=\left[H^{-1}\left(G, \mathbf{C}_{K}\right)\right]=\left[H^{r}\left(G, \mathbf{C}_{K}\right)\right]
$$

On the other hand, since for $a=[\mathbf{A}]=\left[\mathbf{A}:(A)\left(a_{0}\right)\right] \times\left[(A)\left(a_{0}\right):(A)\right]=$ $\left[\mathbf{A}:(A)\left(a_{0}\right)\right] \times a_{0}$ we have $\left[\mathbf{A}:(A)\left(a_{0}\right)\right]=\left[\eta: N E_{K}\right]^{13)}$, we see at once from lemma 1

$$
a=h_{F} \times \frac{I I e(p)}{\left[H^{1}\left(E_{\mathrm{K}}\right)\right]} \times\left[\eta: N E_{\mathrm{K}}\right]
$$

Since $\frac{\left[H^{0}\left(G, E_{K}\right)\right]}{\left[H^{1}\left(G, E_{K}\right)\right]}=Q\left(E_{K}\right)=\frac{\Pi e\left(p_{\infty}\right)}{n}$ by lemma 3, and $\left[H^{0}\left(G, E_{K}\right)\right]=\left[\varepsilon: N E_{K}\right]$ $=[\varepsilon: \eta]\left[\eta: N E_{K}\right]$, we have

$$
a=h_{F} \times \frac{\tilde{\Pi} e(\mathfrak{p})}{n \cdot[\varepsilon: \eta]}
$$

10) Cf. lemma 4.

12) For the absolutely cyclic extension, this relation is already found in S. Iyanaga-

T. Tamagawa [15]. Cf. H. W. Leopoldt [17], too.

12) Cf. lemma 5.

13) Cf. lemma 6. 
Consequently, we obtain $n=n_{1} \times n_{2}$ from (1) and (2), and it is clear from (2) that $h_{w} \times \tilde{\Pi} e(p) \equiv 0 \bmod n$ holds. Thus we have proved all the assertions of our lemma 4.

Lemma 5. $\tilde{\Pi} e(p)$ is divisible by $[\varepsilon: \eta]$ and the conditions:

$$
\text { (I) } a=h_{r}, \quad \text { (II) } \frac{\tilde{n} e(p)}{[\varepsilon: \eta]}=n
$$

are equivalent to each other.

Proof. Let $(\nu)$ be the group of principal ideals $(\nu)$ in $F$ such that $\nu$ is a norm residue of $\bmod$ an ideal $m$ with respect to $K / F$. If we choose the ideal in suitably, then the index of $(\nu)$ in $(\alpha)$ is equal to $\widetilde{\Pi} e(p) /[\varepsilon: \eta]$. Hence $\tilde{\Pi} e(p)$ is divisible by $[\varepsilon: \eta]$.

On the other hand, it is evident from lemma 4 that $a=h_{F}$ and $\widetilde{\Pi} e(p)=n \cdot[\varepsilon: \eta]$ are equivalent to each other.

Lemma 6. In the decomposition

$$
a=[\mathbf{A}]=\left[\mathbf{A}:(A)\left(a_{0}\right)\right]\left[(A)\left(a_{0}\right):(A)\left(a_{F}\right)\right]\left[(A)\left(a_{F}\right):(A)\right]
$$

of $a$, we have $\left[\mathbf{A}:(A)\left(a_{0}\right)\right]=\left[\eta: N E_{K}\right],\left[(A)\left(a_{0}\right):(A)\left(a_{F}\right)\right]=\frac{\Pi e(\mathfrak{p}) \cdot h_{0}}{\left[H^{1}\left(G, E_{K}\right)\right]}$ and $\left[(A)\left(a_{F}\right):(A)\right]=\frac{h_{F}}{h_{0}}$. Hence

$$
a=\left[\eta: N E_{K}\right] \times \frac{\Pi e(\mathfrak{p}) \cdot h_{0}}{\left[H^{1}\left(G, E_{K}\right)\right]} \times \frac{h_{F}}{h_{0}} .
$$

Proof. To any ideal a belonging to an ambiguous class in $K / F$, there corresponds an unit $\eta$ in $(\eta)$ in the following way:

since $a^{1-\sigma}$ is a principal ideal, there exists a number $\theta$ in $K$ such that $a^{1-\sigma}=(\theta)$, and $N \theta$ is clearly an unit $\eta$ in $F$. In this correspondence, an ideal which belongs to an ideal class represented by an ambiguous ideal in $K / F$ corresponds to an element in $N E_{K}$. Hence we have

$$
\left[\mathbf{A}:(A)\left(a_{0}\right)\right]=\left[\eta: N E_{K}\right] \text {. }
$$

$\left[(\mathbf{A})\left(a_{F}\right):(\mathbf{A})\right]=h_{F} / h_{0}$ is evident from the definition of $h_{0}$.

Finally, from the above two assertions and lemma 4 we see easily $\left[(\mathbf{A})\left(\mathfrak{a}_{0}\right):(\mathbf{A})\left(\mathfrak{a}_{F}\right)\right]=\Pi e(\mathfrak{p}) \cdot h_{0} /\left[H^{1}\left(G, E_{R}\right)\right]$. 


\section{$\S 3$. Ideal class group}

We shall, here, consider the relative genus field (Geschlechterkörper). Let $K / F$ be an abelian extension of a number field $F$ of finite degree, and let $K^{*}$ be the maximal extension field which is abelian over $F$ and unramified over $K$. After Hasse-Leopoldt ${ }^{14}$ we shall call such a extension field $K^{*}$ the relative genus field with respect to $K / F$, and call the relative degree $g^{*}=\left[K^{*}: K\right]$ the relative genus number with respect to $K / F$. Moreover, we shall call the ideal group $H^{*}$, to which the relative genus field $K^{*}$ corresponds by class field theory, the relative principal genus with respect to $K / F$.

Proposition 1. If $K / F$ is a cyclic extension of $F$, then the relative principal genus $H^{*}$ with respect to $K / F$ is the $(1-\sigma)$-th power of the ideal class group $\mathbf{C}_{K}$ of $K$, i.e. $H^{*}=\mathbf{C}_{K}^{1-\sigma}$, where $\sigma$ is a generator of the Galois group $G=G(K / F)$. (Relative principal genus theorem)

Moreover, the relative genus number $g^{*}$ with respect to $K / F$ is equal to the ambiguous class number a with respect to $K / F$, i.e. $g^{*}=a$.

Proof. Since $K^{*}$ is an unramified abelian extension over $K, K^{*}$ is contained in the absolute class field of $K$. Hence the relative principal genus $H^{*}$ with respect to $K / F$ contains the group of principal.ideals in $K$ and is composed of ideal classes in $K$. By the criterion of Hasse ${ }^{15}$, the relative principal genus $H^{*}$ must contain the $(1-\sigma)$-th power $\mathbf{C}_{K}^{1-\sigma}$ of the ideal class group $\mathbf{C}_{K}$. Moreover, $H^{*}$ must bé equal to $\mathbf{C}_{K}^{1-\pi}$ because of the maximal property of the relative genus field $K^{*}$.

Next, in the homomorphism of $\mathbf{C}_{K}$ onto $\mathbf{C}_{K}^{1-\sigma}$ the kernel is evidently the group of ambiguous ideal classes $\mathrm{A}$ with respect to $K / F$. Hence from the theorem of homomorphism and the above relation $H^{*}=\mathbf{C}_{K}^{1-a}$, it follows at once that

$$
g^{*}=\left[K^{*}: K\right]=\left[\mathbf{C}_{K}: H^{*}\right]=\left[\mathbf{C}_{K}: \mathbf{C}_{K}^{1-\sigma}\right]=[\mathbf{A}]=a .
$$

Proposition 2. Let $K / F$ be a cyclic extension of degree $n$, and denote by $a_{1}$ the order of $\mathbf{A} \cap \mathbf{C}_{K}^{1-\sigma}$, i.e. $a_{1}=\left[\mathbf{A} \cap \mathbf{C}_{k}^{1-\sigma}\right]$. Then we have

(i) $\mathbf{C}_{K}=\mathbf{A}+\mathbf{C}_{K}^{1-\sigma}$ is direct if and only if $a_{1}=1$,

(ii) $a$ is not prime to the degree $n$ if $a_{1} \neq 1$,

14) Cf. H. Hasse [9] and H. W. Leopoldt [17].

15) Cf. H. Hasse [10], II, $\$ 5$. 
where we denote by o a generator of the Galois group $G=G(K / F)$.

Proof. It is evident from the fact $h_{K}=a \times b_{1}$ that $\mathbf{C}_{K}=\mathbf{A}+\mathbf{C}_{\mathrm{K}}^{1-\sigma}$ is direct if and only if $a_{1}=\left[\mathbf{A} \cap \mathbf{C}_{K}^{1-\sigma}\right]=1$, where $b_{1}=\left[\mathbf{C}_{K}^{1-\sigma}\right]$.

Next, we consider the factor group $\mathbf{B}=\mathbf{C}_{\boldsymbol{K}} / \mathbf{A}$ of the ideal class group $\mathbf{C}_{K}$ modulo the group of ambiguous classes A with respect to $K / F$. Since the group of ambiguous classes $A$ is a $G$-invariant subgroup of $\mathbf{C}_{K}$, the factor group $\mathbf{B}$ is also a $G$-module and $\mathbf{B}$ is isomorphic with the group $\mathbf{C}_{K}^{1-0}$ as $G$ module. Therefore, if $a_{1} \neq 1$, then there exists an element $B \notin \mathbf{A}$ of $\mathbf{B}$ such that $B^{\sigma}=B$ holds. Namely, there exists an ideal class $C$ of $\mathbf{C}_{\mathrm{k}}$ such that $C^{3}=C A$ holds for some ambiguous class $A$ which is not the principal ideal class of $\mathbf{C}_{K}$. Since $C=C^{\sigma^{n}}=C A^{n}, A^{n}$ is the principal ideal class of $\mathbf{C}_{K}$. Hence the order a of the group $\mathbf{A}$ is not prime to $n$.

Proposition 3. Let $K / F$ be a cyclic extension of a prime power degree $l^{\nu}$, and put $a_{i}=\left[\mathbf{A} \cap \mathbf{C}_{\mathbf{K}}^{(1-\sigma)^{t}}\right], b_{j}=\left[\mathbf{C}_{\mathbf{K}}^{(1-\sigma)^{j}}\right](i, j=0,1,2, \ldots)$. Then there exists an integer $s(\geqq 0)$ such that

(i) $h_{K}=a_{0} \times a_{1} \times \cdots \times a_{s-1} \times a_{s} \times b_{s+1}$,

(ii) $a_{i}$ is divisible by $a_{i+1}(i=0,1, \ldots, s-1)$,

(iii) $\left\{\begin{array}{l}a_{0} \equiv a_{1} \equiv \cdots \equiv a_{s-2} \equiv 0 \\ a_{s-1}>a_{s}=1, b_{s+1} \equiv 1\end{array} . \quad(\bmod l)\right.$.

Proof. Since the group $\mathbf{C}_{K}$ is an abelian group with finite order $h_{K}$, there exists an integer $s \geqq 0$ such that $\mathbf{C}_{K} \varsubsetneqq \mathbf{C}_{K}^{1-\sigma} \varsubsetneqq \mathbf{C}_{K}^{(1-\sigma)^{2}} \cdots \cdots \risingdotseq \mathbf{C}_{E}^{(1-\sigma)^{s-1}} \equiv \mathbf{C}_{K}^{(1-\sigma)^{s}}=\mathbf{C}_{K}^{(1-\sigma)^{s+1}}$ $=\cdots$, where $\sigma$ is a generator of the Galois group $G=G(K / F)$.

Put here $\mathbf{A}_{i}=\mathbf{A} \cap \mathbf{C}_{K}^{(1-a)^{t}}$ for convenience. Then, since $b_{i}=a_{i} \times b_{i+1}(i=0$, $1,2, \ldots)$ and

$$
\mathbf{A}_{0}=\mathbf{A} \supseteqq \mathbf{A}_{1} \supseteqq \mathbf{A}_{2} \supseteqq \cdots \supseteqq \mathbf{A}_{s-1}
$$

we have first

$$
h_{K}=b_{0}=a_{0} \times b_{1}=a_{0} \times\left(a_{1} \times b_{2}\right)=\cdots=\left(\Pi_{i=0}^{s} a_{i}\right) \times b_{s+1}
$$

and $a_{s-1} \neq a_{s}=1$.

Next, since each $\mathbf{A}_{i+1}$ is a subgroup of $\mathbf{A}_{i}, a_{i}$ is divisible by $a_{i+1}$ for every integer $i=0,1,2, \ldots, s-1$.

Finally, since $\left[\mathbf{A} \cap \mathbf{C}_{K}^{(1-\sigma)^{s-1}}\right]=a_{s-1} \neq 1$ holds, we know easily by the same way as in the proof of proposition 2 that the order $a_{s-2}$ of $\mathrm{A} \cap \mathbf{C}_{K}^{(1-3)^{s-2}}$ is not. 
prime to the degree $l^{\nu}$ of $K / F$, namely $a_{s-2}$ is divisible by $l$. Therefore we get $a_{0} \equiv a_{1} \equiv \cdots \equiv a_{s-2} \equiv 0$ mod. $l$. Since the order of the Galois group $G=G(K / F)$ is a prime power $l^{\nu}$, each element of $\mathbf{C}_{K}^{(1-0)^{i}}$ which is not in $\mathbf{A} \cap \mathbf{C}_{K}^{(1-\sigma)^{i}}$ has at least two, and so a multiple of the prime $l$ different $G$-conjugates for every $i=0,1, \ldots$ Therefore we have at once $b_{i} \equiv a_{i}$ mod. $l$ in the decomposition of $b_{i}$, i.e. $b_{i}=a_{i} \times b_{i+1}$. In particular, since $a_{s}=1$ we have $b_{s+1}=a_{s} \times b_{s+1}=b_{s} \equiv$ $a_{s}=1$ mod. $l$.

\section{§ 4. Ideal class number and unit group}

Proposition 4. Let $K / F$ be any Galois extension of finite degree $n$. If $h_{F}$ is prime to the degree $n$, i.e. $\left(h_{F}, n\right)=1$, then

(i) $\mathbf{A}_{F}=(A)\left(\mathfrak{a}_{F}\right) \cong \mathbf{C}_{F}$ i.e. $h_{F}=\left[\mathbf{A}_{F}:(A)\right]$

and $h_{0}=n_{1}=1$,

(ii) $\mathbf{C}_{K}=\mathbf{A}_{F}+{ }_{N} \mathbf{C}_{K}$ is direct,

(iii) $\Pi e(\mathfrak{p})=\left[H^{1}\left(G, E_{K}\right)\right]\left[(A)\left(\mathfrak{a}_{0}\right):(A)\left(\mathfrak{a}_{F}\right)\right]$.

Proof. (i) By the assumption $\left(h_{F}, n\right)=1$ and lemma 2, 4, we have $h_{0}=1$, $n_{1}=1$ at once. Hence we obtain $h_{F}=\left[\mathbf{A}_{F}:(A)\right]$ and a natural isomorphism $\mathbf{A}_{F} \cong \mathbf{C}_{F}$.

(ii) Let $C$ be any ideal class in $A_{F} \cap{ }_{N} \mathbf{C}_{R}$. Then, since $C$ belongs to ${ }_{N} \mathbf{C}_{E}$. $N_{K / F} C$ is the principal ideal class $I_{F}$ in $\mathbf{C}_{F}$. Moreover, since $C$ also belongs to $\mathbf{A}_{F}$, we have $N_{K / F} C=a_{F}^{n} \cdot I_{F}$ for an ideal $a_{F}$ in $F$. Hence $a_{F}^{n}$ is a principal ideal of $F$. On the other hand, from the assumption $\left(h_{F}, n\right)=1, a_{F}$ itself must be a principal ideal of $F$. Hence $C$ is the principal ideal class of $\mathbf{C}_{K}$, namely $\mathbf{A}_{F}+{ }_{N} \mathbf{C}_{K}$ is direct in $\mathbf{C}_{\boldsymbol{K}}$.

Next, since $h_{F}$ is prime to $n, \mathbf{A}_{F}$ is isomorphic to $\mathbf{C}_{F}$ and $N_{K / F} \mathbf{A}_{F}=\mathbf{C}_{F}$ holds. Hence we obtain $N_{K / K} \mathbf{C}_{K}=N_{K / F} \mathbf{A}_{F}=\mathbf{C}_{F}$. Thus we know that $\mathbf{C}_{K}$ is contained in $A_{F}+{ }_{\Delta} \mathbf{C}_{K}$, namely we know that $\mathbf{C}_{K}=A_{F}+{ }_{N} \mathbf{C}_{K}$ is direct.

(iii) By proposition 4, (i) we have $a_{0}=h_{F} \cdot\left[(A)\left(a_{0}\right):(A)\left(a_{F}\right)\right]$. Hence we have $\Pi e(p)=\left[H^{1}\left(G, E_{\kappa}\right)\right]\left[(A)\left(\mathfrak{a}_{0}\right):(A)\left(\mathfrak{a}_{k}\right)\right]$ by lemma 1 .

Proposition 5. If $K / F$ is a cyclic extension of finite degree $n$ and $a$ is prime to the degree $n$, i.e. $(a, n)=1$, then we have

(i) $\mathbf{C}_{K}=\mathbf{A}+\mathbf{C}_{K}^{1-\sigma}$ is direct,

(ii) $a=h_{F} / h_{0}, h_{0}=n_{1}$ and $a_{1}=1$,

(iii) $\left[H^{1}\left(G, E_{K}\right)\right]=\Pi e(p) \cdot h_{0},\left[H^{0}\left(G, E_{K}\right)\right]=[\varepsilon: \eta]=h_{0} \cdot \widetilde{\Pi} e(p) / n, H^{r}\left(G, \mathbf{C}_{K}\right)$ 
$=\{1\}$ for any integer $r$.

Moreover, if we assume that $K / F$ is cyclic with a prime power degree $l^{\nu}$, then we have $b_{1}=b_{2} \equiv 1$ mod. $l$, where $b_{i}=\left[\mathbf{C}_{K}^{(1-\sigma)^{i}}\right](i=1,2)$.

Remark. The natural homomorphism $\mathbf{C}_{F} \rightarrow \mathbf{C}_{K}$ gives an isomorphism of $N \mathbf{C}_{K} \subset \mathbf{C}_{F}$ into $\mathbf{C}_{K}$. For, since $\left[N \mathbf{C}_{K}:(\alpha)\right]=h_{F} / n_{1},\left[(A)\left(a_{F}\right):(A)\right]=h_{F} / h_{0}=[\mathbf{A}]$ and $h_{0}=n_{1}$ by proposition 5 , (ii), we have $\left[N \mathbf{C}_{K}:(\alpha)\right]=\left[(A)\left(\mathfrak{a}_{F}\right):(A)\right]$.

Proof. By the assumption $(a, n)=1$ and proposition 2 , we know that $a_{1}=1$ and $\mathbf{C}_{K}=\mathbf{A}+\mathbf{C}_{K}^{1-0}$ is direct. In particular, we have $b_{1}=b_{2} \equiv 1$ mod. $l$ by proposition 3 provided that $K / F$ is cyclic with a prime power degree $l^{\nu}$.

On the other hand, the numbers

$$
\frac{h_{F}}{n_{1}}, \frac{\tilde{\eta} e(p)}{n_{2} \cdot[\varepsilon: \eta]},\left[\eta: N E_{K}\right], \frac{\Pi e(p)}{\left[H^{1}\left(G, E_{K}\right)\right]} \cdot h_{0} \text { and } \frac{h_{F}}{h_{0}}
$$

appearing in the representations

$$
a=\frac{h_{F}}{n_{1}} \times \frac{\tilde{n} e(\mathfrak{p})}{n_{2} \cdot[\varepsilon: \eta]}=\left[\eta: N E_{K}\right] \cdot \frac{\Pi e(p)}{\left[H^{1}\left(G, E_{K}\right)\right]} \cdot h_{0} \times \frac{h_{F}}{h_{0}} \text { of } \mathrm{a},
$$

are all integers. Moreover $\frac{\widetilde{\Pi} e(p)}{n_{2} \cdot[\varepsilon: \eta]},\left[\eta: N E_{K}\right], \frac{\Pi e(p)}{\left[H^{1}\left(G, E_{K}\right)\right]} \cdot h_{0}$ are composed of the prime factors of $n$. Hence we have $\frac{\widetilde{I} e(p)}{n_{2} \cdot[\varepsilon: \eta]}=\left[\eta: N E_{K}\right]=$ $\frac{\Pi e(\mathfrak{b})}{\left[H^{1}\left(G, E_{K}\right)\right]} \cdot h_{0}=1$ and $\left[H^{1}\left(G, E_{K}\right)\right]=\Pi e(\mathfrak{p}) \cdot h_{0},\left[H^{0}\left(G, E_{K}\right)\right]=[\varepsilon: \eta]=\tilde{\Pi} e(\mathfrak{p}) / n_{2}$. Furthermore we have $a=h_{F} / h_{0}=h_{F} / n_{1}$. Therefore we obtain $h_{0}=n_{1}$ and hence $\left[H^{0}\left(G, E_{K}\right)\right]=\widetilde{\Pi} e(p) / n_{2}=\widetilde{\Pi} e(p) \cdot h_{0} / n$.

\section{$\S 5$. Main theorems}

Theorem 1. Let $K / F$ be a finite extension over a number field $F$ of finite degree such that $K$ and the absolute class field $\widetilde{F}$ of $F$ are disjoint over $F$, i.e. $\widetilde{F} \cap K=F$. Then we have

(i) if $K / F$ is Galois, then $h_{K}$ is divisible by $h_{F}$, i.e. $h_{F} / h_{K}$,

(ii) if $K / F$ is abelian, then the relative genus number $g^{*}$ with respect to $K / F$ is divisible by $h_{F}$, i.e. $h_{F} / g^{*}$,

(iii) if $K / F$ is cyclic, then $a$ is divisible by $h_{F}$, i.e. $h_{F} / a$,

(iv) if $K / F$ is cyclic and has one and only one ramified prime divisor, then $h_{F}$ is equal to $a$ and $[\varepsilon: \eta]=1$. 
Proof. (i) This assertion is already known ${ }^{16)}$, but for the sake of com. pleteness, we add a simple proof.

Since $\widetilde{F} K / K$ is unramified and its Galois group $G(\widetilde{F} K / K)$ is isomorphic to the Galois group $G(\widetilde{F} / F), \widetilde{F} K$ is contained in the absolute class field $\widetilde{K}$ of $K$ and the relative degree $[\widetilde{F} K: K]$ is equal to the relative degree $[\widetilde{F}: F]=h_{F}$. Hence $h_{K}$ is divisible by $h_{F}$.

(ii) Since $\widetilde{F} K / K$ is unramified and $\widetilde{F} K / F$ is abelian, $\widetilde{F} K$ is contained in the relative genus field $K^{*}$ with respect to $K / F$. Therefore the relative genus number $g^{*}$ with respect to $K / F$ is divisible by $[\widetilde{F} K: K]=[\widetilde{F}: F]=h_{F}$.

(iii) Since by proposition 1 the number a of ambiguous ideal classes with respect to $K / F$ is equal to the relative genus number $g^{*}$ with respect to $K / F$, our assertion (iii) is obvious by (ii).

(iv) By the above proved (ii) and lemma $4, a / h_{F}=\tilde{\Pi} e(p) /[K: F][\varepsilon: \eta]$ is a rational integer. On the other hand, from the assumption that $K / F$ has one and only one ramified prime divisor and $\widetilde{F} \cap K=F$, we have at once $\widetilde{\Pi} e(p)=[K: F]$. Hence we obtain $[\varepsilon: \eta]=1$ and $a / h_{F}=1$.

Theorem 2. Let $K / F$ be a cyclic extension of a finite degree $n$. If we assume $a=h_{F}$, then we have

(i) $\tilde{\Pi} e(\mathfrak{p})=n \cdot[\varepsilon: \eta]$,

(ii) $\left[H^{1}\left(G, E_{K}\right)\right]=\Pi e(p) \cdot\left[\eta: N E_{K}\right]$,

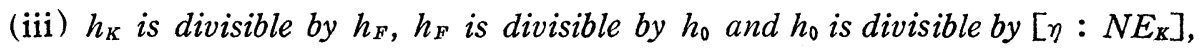
i.e. $\left[\eta: N E_{K}\right] / h_{0} / h_{F} / h_{K}$.

Furthermore, if we assume that $K / F$ is cyclic with a prime power degree $l^{2}$, then $h_{F}$ is not prime to $l$ provided that $h_{K}$ is not prime to $l$.

Proof. (i) This assertion follows trivially from lemma 5 and assumption $a=h_{F}$.

(ii) By lemma 3 and (i) we have easily

$$
\begin{aligned}
& {\left[H^{1}\left(G, E_{K}\right)\right]=\frac{n \cdot\left[H^{0}\left(G, E_{K}\right)\right]}{\Pi e\left(\mathfrak{p}_{\infty}\right)}=\frac{n \cdot[\varepsilon: \eta]\left[\eta: N E_{K}\right]}{\Pi e\left(\mathfrak{p}_{\infty}\right)}=\frac{\tilde{\Pi} e(\mathfrak{p})\left[\eta: N E_{K}\right]}{\prod e\left(\mathfrak{p}_{\infty}\right)}} \\
& =\Pi e(p)\left[\eta: N E_{k}\right] \text {. }
\end{aligned}
$$

(iii) Since $h_{K}=a \times b_{1}$ is divisible by $a=h_{F}$, we know first $h_{F} / h_{K}$. Next, $h_{0} / h_{F}$ is evident from lemma 2. Finally, from lemma 6 and theorem 2, (ii),

16) Cf. e.g. C. Chevalley [4], K. Iwasawa [12] or N. C. Ankeny-S. Chowla-H. Hasse [1]. 
it follows that $\left[(A)\left(a_{0}\right):(A)\left(a_{F}\right)\right]=\Pi e(p) \cdot h_{0} /\left[H^{1}\left(G, E_{K}\right)\right]=h_{0} /\left[\eta: N E_{\mathrm{K}}\right]$ is integer, and so $\left[\eta_{\eta}: N E_{K}\right] / h_{0}$.

Moreover, we assume that $K / F$ is cyclic with a prime power degree $l^{\nu}$. If $h_{F}$ is prime to $l$, then by the assumption $a=h_{F}, a$ is prime to $l$. Hence we have $b_{1} \equiv 1$ mod. $l$ by proposition 5 .

Since $h_{K}=a \times b_{1}$, we know that $h_{K}$ is prime to $l$ provided $h_{F}$ is prime to $l$.

It is evident that those theorems 1,2 are a generalization of the theorem of $\mathrm{K}$. Iwasawa.

Next, we give a corollary of this theorem 2 which is a generalization of the result of S. $-\mathrm{N}$. Kuroda ${ }^{17)}$ for a cyclic extension of prime degree.

Corollary. Let $K / F$ be a cyclic extension of finite degree $n$ and denote by $\sigma$ a generator of the Galois group $G=G(K / F)$. If we assume that $a=h_{F}$ and $h_{F}$ is prime to $n$, then we have

(i) $a=a_{0}=h_{F}, a_{1}=h_{0}=n_{1}=1$,

(ii) $\mathbf{C}_{K}=\mathbf{A}+\mathbf{C}_{\mathbf{K}}^{1-\sigma}=\mathbf{A}_{F}+{ }_{\Lambda} \mathbf{C}_{K}$ (direct),

(iii) $\left[\eta: N E_{K}\right]=1,\left[H^{0}\left(G, E_{K}\right)\right]=[\varepsilon: \eta]=\tilde{I} e(p) / n,\left[H^{1}\left(G, E_{K}\right)\right]=\Pi e(p)$, $H^{r}\left(G, \mathbf{C}_{K}\right)=\{1\}$ for every integer $r$.

Moreover, if we assume that $K / F$ is cyclic with a prime power degree $l^{\nu}$, then we have

(iv) $h_{K}$ is prime to $l$,

(v) $b_{1}=h_{K} / h_{F} \equiv 1 \bmod$. $l$.

Proof. This corollary is evident by theorem 2 and proposition 4,5 .

\section{Appendix. Unramified cyclic extension.}

In this appendix we shall consider an unramified cyclic extension $K / F$ over an algebraic number field $F$ of finite degree. Namely, we prove the following proposition :

Proposition. Let $K / F$ be an unramified cyclic extension, then we have

(i) $[\varepsilon: \eta]=1$, i.e. $\left[H^{0}\left(G, E_{K}\right)\right]=\left[\eta: N E_{K}\right]$,

(ii) $a=h_{F} /[K: F]{ }^{18)}$ i.e. $\widetilde{F}=K^{*}$,

(iii) $h_{0}=\left[H^{1}\left(G, E_{K}\right)\right]=[K: F]\left[\eta: N E_{K}\right]$,

17) Cf. S.-N. Kuroda [16]

18) For the cyclic extension of prime degree, this relation is already found in $M$. Moriya [18], T. Honda [11] etc. 
where $G=G(K / F)$ is the Galois group of $K / F$, and $K^{*}$ is the relative genus field with respect to $K / F$.

Remark. Assertion (iii) says that the number $h_{0}$ of ideal classes of $F$ which become principal in $K$ is a multiple of the degree $[K: F]$, and that the principal ideal theorem of Terada-Tannaka ${ }^{191}$ claiming that all the ambiguous ideal classes with respect to $K / F$ become principal in the absolute class field $\widetilde{F}$ of $F$ is truely a generalization of the original principal ideal theorem of Hilbert-Furtwängler ${ }^{201}$ when $\left[H^{0}\left(G, E_{K}\right)\right]=\left[\eta: N E_{K}\right] \neq 1$. For, by assertion (iii), $\left[\eta: N E_{K}\right]=1$ holds if and only if $h_{0}=[K: F]$, and moreover by the assertion (ii) the condition $h_{0}=[K: F]$ is equivalent to $a=h_{F} / h_{0}$. On the other hand, the relation $a=h_{F} / h_{0}$ is equivalent to $\left[\mathbf{A}:(A)\left(a_{F}\right)\right]=1$ by lemma 6 , namely the group of ambiguous ideal classes $\mathbf{A}$ with respect to $K / F$ is exactly the group of ideal classes of $K$ represented by ideals of $F$.

Proof. (i) Since $K / F$ is an unramified cyclic extension, $[\varepsilon: \eta]=1$ is evident from lemma 5.

(ii) From (i) and lemma 4 we obtain at once $a=h_{F} /[K: F]$. Hence we have easily $\widetilde{F}=K^{*}$ by proposition 1 .

(iii) Since $K / F$ is unramified, we have $a_{0}=h_{F} /\left[H^{1}\left(G, E_{K}\right)\right]$ by lemma 1 and $a_{n}=\left[(A)\left(a_{F}\right):(A)\right]$ from the definition of $a_{0}$, respectively. On the other hand, we have $\left[(A)\left(\mathfrak{a}_{F}\right):(A)\right]=h_{F} / h_{0}$ by lemma 6 . Hence we obtain $h_{0}=$ $\left[H^{1}\left(G, E_{K}\right)\right]$ for any unramified extension $K / F$. In particular, if $K / F$ is cyclic and unramified, then we obtain moreover $\left[H^{1}\left(G, E_{K}\right)\right]=[K: F]\left[\eta: N E_{K}\right]$ by lemma 3 and assertion (i).

\section{REFERENCES}

[1] N. C. Ankeny-S. Chowla-H. Hasse, On the class-number of the maximal real subfield of a cyclotomic field, J. reine angew. Math., 217 (1965), 217-220.

[2] E. Artin, Über Einheiten relativ Galoischer Zahlkörper, J. reine angew. Math., 167 (1931), 153-156.

[3] A. Brumer-M. Rosen, Class number and ramification in number fields, Nagoya Math. J., 23 (1963), 97-101.

[4] C. Chevalley, Relation entre le nombre de classes d'un sous-corps et celui d'un surcorps, C. R. Sci. Paris, 192 (1931), 257-258.

19) Cf. F. Terada [20] and T. Tannaka [19].

20) $\mathrm{Cf}$. $\mathrm{Ph}$. Furtwängler [8] etc. 
[ 5 ] C. Chevalley, Class field theory. (Th. 10.3), Notes at Nagoya University 1954.

[6] Ph. Furtwängler, Über die Klassenzahlen abelscher Zahlkörper, J. reine angew. Math., 134 (1908), 91-94.

[7] Ph. Furtwängler, Über die Klassenzahlen der Kreisteilungskörper, J. reine angew. Math., 140 (.1911), 29-32.

[8] Ph. Furtwängler, Beweis des Hauptidealsatzes für die Klassenkörper algebraischer Zahlkörper, Abh. Math. Sem. Hamburg, 7 (1930), 14-36.

[ 9 ] H. Hasse, Zur Geschlechtertheorie in quadratischen Zahlkörper, J. Math. Soc. Japan., 3 (1951), 45-51.

[10] H. Hasse, Bericht über neuere Untersuchungen und Probleme aus der Theorie der algebraischen Zahlkőrper II, (1930), Jahresberichte der D.M.V.

[11] T. Honda, On absolute class fields of certain algebraic number field, J. reine angew. Math., 203 (1960), 80-89.

[12] K. Iwasawa, A note on class numbers of algebraic number fields, Abh. Math. Sem. Hamburg, 20 (1956), 257-258.

[13] K. Iwasawa, A note on the group of units of algebraic number field, J. math. pure appl., 35 (1956), 189-192.

[14] K. Iwasawa, A class number formula for cyclotomic fields, Ann. of Math., 76 (1962), 171-179.

[15] S. Iyanaga-T. Tamagawa, Sur la théorie du corps de classes sur le corps de nombres rationelles, J. Math. Soc. Japan, 3 (1951), 220-227.

[16] S.-N. Kuroda, Über die Klassenzahl eines relativzyklischen Zahlkörpers von Primzahlgrade, Proc. Japan Acad., 40 (1964), 623-626.

[17] H. W. Leopoldt, Zur Geschlechtertheurie in abelschen Zahlkörpern, Math. Nachr., 9 (1953), 351-362.

[18] M. Moriya, Über die Klassenzahl eines relativzyklischen Zahlkőrpern von Primzahlgrad, Japanese J. Math., 10 (1933), 1-18.

[19] T. Tannaka, Some remarks concerning principal ideal theorem, Töhoku Math. J., 1 (1949), 270-278.

[20] F. Terada, On a generalization of the principal ideal theorem, Tōhoku Math. J., 1 (1949), 229-269.

[21] H. Weber, Theorie der algebraischen Zahlkörper, Acta Math., 8 (1886), 193-263.

\section{Mathematical Institute}

Nagoya University 